\title{
Classification of Perturbations for Membranes with Bending Rigidity
}

\author{
Kay Jörg Wiese年 \\ CEA, Service de Physique Théorique, CE-Saclay \\ F-91191 Gif-sur-Yvette Cedex, FRANCE
}

\begin{abstract}
A complete classification of the renormalization-group flow is given for impuritylike marginal operators of membranes whose elastic stress scales like $(\Delta r)^{2}$ around the external critical dimension $d_{c}=2$. These operators are classified by characteristic functions on $\mathbb{R}^{2} \times \mathbb{R}^{2}$.
\end{abstract}

\footnotetext{
*Email: wiese@amoco.saclay.cea.fr
} 


\section{Introduction}

Fluctuating tethered membranes have attracted much interest during the last years. Considerable theoretical advance has been made through the work of F. David, B. Duplantier and E. Guitter [1] who proved that the theory described by

$$
\mathcal{H}=\int \mathrm{d}^{D} x \frac{1}{2} r(x)(-\Delta)^{k / 2} r(x)+\lambda \delta^{d}(r(x))
$$

with $k \geq 2$ is a renormalizable field theory, if $D$ and $d$ are properly chosen. The case $k=2$ corresponds to the case of a $D$-dimensional Gaussian manifold imbedded in $d$ dimensions. The field

$$
r: x \in \mathbb{R}^{D} \longrightarrow r(x) \in \mathbb{R}^{d}
$$

is the coordinate of the membrane.

For $k=4$ (1) represents a manifold with vanishing tension but with bending rigidity. In this case $r(x)$ is the amplitude of the orthogonal modes, the membrane thus imbedded in $D+d$ dimensions. It is this latter object which shall be studied in the following.

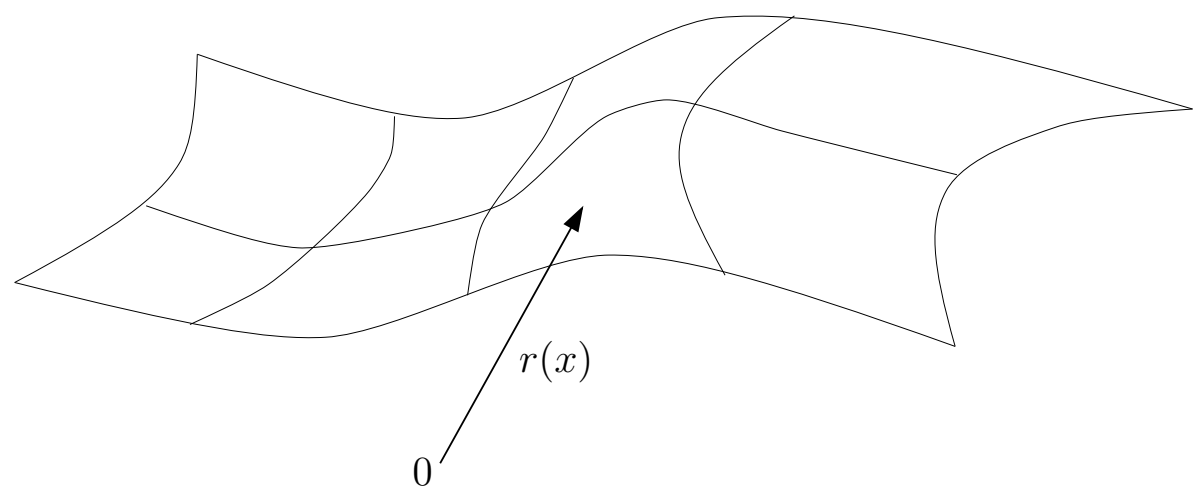

Figure 1: Membrane interacting with a point

The $\delta$-potential describes the interaction of the manifold with a fixed point.

The case of a membrane $(D=2)$ is remarkable as $r$ has dimension -1 in internal momentum-units such that $\nabla r$ is dimensionless. Possible marginal perturbations are thus

$$
\mathcal{H}_{\text {int }}=\int \mathrm{d}^{D} x \delta^{d}(r(x)) f(\nabla r(x))
$$

with an arbitrary function $f$ instead of a simple $\delta$-distribution. The model has infinite many marginal perturbations for $D=d=2$ and one expects a rich mathematical structure. The goal is to find the eigen-operators of the renormalization-group flow.

The paper is organized as follows: First a brief description of the model without interaction is given. It is shown that it is not conformal invariant. Therefore the methods of conformal field theory do not apply and the model can only be studied in the framework of perturbation-theory. After reviewing the relation between the 1-loop $\beta$-function and the leading coefficient in the operator product expansion the eigen-operators are constructed. The renormalization-group flow and the physical relevance are discussed. 


\section{Description of the free model}

We are interested in membranes $(D=2)$, whose motion is governed by bending rigidity. Let us therefore introduce the free Hamiltonian $\left(r: \mathbb{R}^{D} \rightarrow \mathbb{R}^{d}\right)$

$$
\mathcal{H}_{0}=-\frac{1}{2(4-D)(2-D)} \int_{x} \frac{1}{2}(\Delta r(x))^{2}
$$

where we abbreviated the integration measure $\left(S_{D}\right.$ is the volume of the $D$-dimensional unit-sphere)

$$
\int_{x}=\frac{1}{S_{D}} \int \mathrm{d}^{D} x, \quad S_{D}=2 \frac{\pi^{D / 2}}{\Gamma(D / 2)}
$$

At the end of the calculations we intend to take the limit $D \rightarrow 2$. The factor $\frac{1}{2-D}$ therefore seems to be rather strange. It is however necessary to define an analytic continuation of the model for $D \leq 2$. With this choice of the Hamiltonian we have

$$
\frac{1}{2}\left\langle\left(r_{i}(x)-r_{j}(y)\right)^{2}\right\rangle_{0}=\delta_{i j}|x-y|^{4-D},
$$

thus especially

$$
\left\langle(r(x)-r(y))^{2}\right\rangle_{0} \geq 0
$$

as demanded from physical arguments even for $D<2$. The factor $1 /\left(2(4-D) S_{D}\right)$ in front of the action (田) is introduced for pure convenience, i.e. to have normalization 1 in (6).

For $D>2$ the model is positive definite, for $D<2$ negative definite. In the latter regime we understand it as analytical continuation from $D>2$. This phenomenon reflects the fact that the expression for the free 2-point correlation function

$$
\frac{1}{2}\left\langle(r(x)-r(y))^{2}\right\rangle_{0}=2(4-D)(2-D) S_{D} D \int \frac{\mathrm{d}^{D} p}{(2 \pi)^{D}} \frac{\mathrm{e}^{i p x}-1}{p^{4}}
$$

becomes IR-divergent in the limit $D \rightarrow 2$ from above.

\section{Remark about conformal invariance}

An interesting question arising in this context is, whether the 2-dimensional biharmonic model is conformal invariant. Its free Hamiltonian is (with a change in normalization and for a scalar field for simplicity)

$$
S_{0}=\frac{1}{2} \int d^{2} x(\Delta \varphi)^{2}
$$

To answer the question, the stress tensor has to be calculated. It is well known that it is not uniquely defined. We only give the result for one of the symmetric versions of the stress tensor:

$$
T_{\mu \nu}=-\delta_{\mu \nu}\left(\frac{1}{2}(\Delta \varphi)^{2}+\partial_{\rho}\left(\varphi \partial_{\rho} \Delta \varphi\right)\right)+\partial_{\mu} \partial_{\nu} \varphi \Delta \varphi+\varphi \partial_{\mu} \partial_{\nu} \Delta \varphi
$$


We have proven that it is impossible to render the stress tensor both symmetric and traceless. The trace of (10) is:

$$
\Theta=-2 \partial_{\mu}\left(\varphi \partial_{\mu} \Delta \varphi\right)+\varphi \Delta^{2} \varphi
$$

The last term on the r.h.s. is a redundant operator which can be neglected because of the classical equation of motion:

$$
\Delta^{2} \varphi=0
$$

So the trace of the stress tensor has the form

$$
\Theta=-\partial_{\mu} K^{\mu}
$$

where $K^{\mu}$ cannot be written as a total divergence (up to redundant operators). According to [3] this implies that the free theory is scale invariant but not conformal invariant. The standard methods of 2-dimensional conformal field theories thus can not be applied.

It is interesting to note that it is possible to construct a biharmonic conformal field theory by introducing an additional gauge field, which cancels the unwanted terms in the stress tensor [4].

\section{Renormalization and operator product expansion}

Before actually analyzing possible marginal perturbations, let us discuss how these perturbations generate divergencies and how these divergencies have to be treated in the framework of renormalization [6].

The goal of renormalization is to eliminate UV-divergences, occurring in the perturbation expansion of IR-finite physical quantities

$$
\langle\mathcal{O}\rangle_{\lambda_{0}}=\frac{\int D[r] \mathcal{O} \mathrm{e}^{-\mathcal{H}_{0}-\lambda_{0} \mathcal{H}_{\mathrm{int}}}}{\int D[r] \mathrm{e}^{-\mathcal{H}_{0}-\lambda_{0} \mathcal{H}_{\text {int }}}} .
$$

$\mathcal{O}$ e.g. may be a neutral product of vertex-operators

$$
\mathcal{O}=\prod_{n} \mathrm{e}^{i k_{n} r\left(x_{n}\right)} \text { with } \sum_{n} k_{n}=0 .
$$

Denoting the perturbations by

$$
\mathcal{H}_{\text {int }}=\int_{x} E(x),
$$

where $E(x)$ is some local functional of $r(x)$, the $n$-th order term in the perturbative expansion of $\langle\mathcal{O}\rangle_{\lambda_{0}}$ becomes:

$$
\frac{\left(-\lambda_{0}\right)^{n}}{n !} \int_{x_{1}} \ldots \int_{x_{n}}\left\langle\mathcal{O} E\left(x_{1}\right) \ldots E\left(x_{n}\right)\right\rangle^{\mathrm{conn}}
$$

Use was made of the standard abbreviations

$$
\begin{aligned}
\langle\mathcal{A} B\rangle^{\mathrm{conn}} & =\langle\mathcal{A} B\rangle-\langle\mathcal{A}\rangle\langle\mathcal{B}\rangle \\
\langle\mathcal{A} B C\rangle^{\mathrm{conn}} & =\langle\mathcal{A} B C\rangle-\langle\mathcal{A}\rangle\langle\mathcal{B} C\rangle-\langle\mathcal{B}\rangle\langle\mathcal{A} C\rangle-\langle\mathcal{C}\rangle\langle\mathcal{A} B\rangle+2\langle\mathcal{A}\rangle\langle\mathcal{B}\rangle\langle\mathcal{C}\rangle .
\end{aligned}
$$


Let us suppose that UV-divergencies occur according to the operator product expansion for $|x-y| \rightarrow 0, z=\frac{x+y}{2}$ :

$$
E(x) E(y)=\frac{1}{|x-y|^{D-\varepsilon}} E(z)+\text { less singular terms } .
$$

$\varepsilon$ is a small dimensional regularization parameter, which will be defined later. We will prove that the divergences which appear for small $|x-y|$ are of this type. According to 11, 2] these are the only divergencies which may occur. In the perturbation expansion, the first divergent term is

$$
\frac{\lambda_{0}^{2}}{2} \int_{x} \int_{y}\langle\mathcal{O} E(x) E(y)\rangle^{\text {conn }}=\frac{\lambda_{0}^{2}}{2} \int_{z}\langle\mathcal{O} E(z)\rangle \int_{x-y} \frac{1}{|x-y|^{D-\varepsilon}}+\text { less singular terms }
$$

In the last integral the small positive parameter $\varepsilon$ plays the role of an regulator. An IR-cutoff $L$ is also needed. (For the regularization procedure cf. [5].) We get:

$$
\int_{|x-y|<L} \frac{1}{|x-y|^{D-\varepsilon}}=\int_{0}^{L} \frac{\mathrm{d} s}{s} s^{\varepsilon}=\frac{L^{\varepsilon}}{\varepsilon}
$$

At 1-loop order the theory is thus renormalized by introducing a renormalized coupling constant

$$
\lambda=Z^{-1} \mu^{-\varepsilon} \lambda_{0}
$$

where $Z$ takes the form

$$
Z=1+\frac{\lambda}{2 \varepsilon}
$$

This is the only necessary renormalization. Especially the field $r(x)$ has not to be renormalized as is known from [1]. Intuitively this is understood from the observation that no renormalization is needed if the membrane is far away from the origin as in this case the membrane is non-interacting. Thus divergencies are always proportional to operators localized at $r=0$.

The renormalization-group $\beta$-function describes as usual the variation of the coupling constant $\lambda$ with respect to a variation of the renormalization-scale $\mu$ :

$$
\begin{aligned}
\beta(\lambda) & =\left.\mu \frac{\partial}{\partial \mu}\right|_{\lambda_{0}} \lambda \\
& =-\varepsilon \lambda+\frac{1}{2} \lambda^{2}+\mathcal{O}\left(\lambda^{3}\right)
\end{aligned}
$$

For $\varepsilon>0$ this equation has a non-trivial IR-stable fixed point

$$
\lambda^{*}=2 \varepsilon
$$

\section{Perturbations}

Let us analyze the canonical scaling dimensions of the free model in order to determine all marginal perturbations. In internal units such that $[x]=-1$ we have: 


$$
[r]=\frac{D-4}{2}
$$

Therefore

$$
[\nabla r]=\frac{D-2}{2}
$$

and is dimensionless in $D=2$.

Regarding polynomial operators, the following marginal perturbations are possible:

$$
H_{\mathrm{pol}}=\int_{x}(\nabla \nabla r)^{2} f(\nabla r)
$$

where we did not specify the index structure for $\nabla$ and $f$ is an arbitrary function. This is a class of perturbations, we do not want to consider here. This is consistent as they are not generated in perturbation theory. We will see that below. On the other hand we may have impurity-like interactions:

$$
H_{\mathrm{int}}=\int_{x} \tilde{\delta}^{d}(r(x))
$$

which are dimensionless, if

$$
d=\frac{2 D}{4-D}
$$

i.e. for $D=2$, if

$$
d=2 .
$$

We again use convenient normalizations

$$
\tilde{\delta}^{d}(r(x))=(4 \pi)^{d / 2} \delta^{d}(r(x))=\int_{p} \mathrm{e}^{i p r(x)}
$$

with

$$
\int_{p}=\pi^{-d / 2} \int \mathrm{d}^{d} p
$$

to have

$$
\int_{p} \mathrm{e}^{-p^{2} a}=a^{-d / 2} .
$$

The marginal perturbations for $D=2$ and $d=2$ are:

$$
\int_{x}: \mathrm{e}^{i \alpha_{i}^{\mu} \nabla_{\mu} r^{i}(x)} \tilde{\delta}^{d}(r(x)):
$$

Normal-ordering has been used to eliminate contributions due to self-contractions. Let us further introduce the notation of vertex-operators

$$
V_{\alpha k}(x)=: \mathrm{e}^{i \alpha \nabla r(x)} \mathrm{e}^{i k r(x)}:
$$

where the indices for $\alpha$ from (36) have been suppressed. The marginal perturbations now read:

$$
V_{\alpha}(x)=\int_{k} V_{\alpha k}(x)=: \mathrm{e}^{i \alpha \nabla r(x)} \tilde{\delta}^{d}(r(x)):
$$


In the spirit of [2] all possible contractions of perturbations have to be analyzed. At 1-loop order there is only one possibility:

$$
V_{a}(x) V_{\beta}(y)
$$

Following [2], these operators are contracted according to $\left(x-y \rightarrow 0\right.$ and $\left.z=\frac{x+y}{2}\right)$ :

$$
\begin{aligned}
\int_{k} \int_{l} V_{\alpha k}(x) V_{\beta l}(y) & =\int_{k} \int_{l}: V_{\alpha k}(x) V_{\beta l}(y): \mathrm{e}^{-\langle(\alpha \nabla+k) r(x)(\beta \nabla+l) r(y)\rangle_{0}} \\
& =\int_{k} \int_{l}: \mathrm{e}^{(x-z) \partial_{z}} V_{\alpha k}(z) \mathrm{e}^{(y-z) \partial_{z}} V_{\beta l}(z): \mathrm{e}^{-\langle(\alpha \nabla+k) r(x)(\beta \nabla+l) r(y)\rangle_{0}}
\end{aligned}
$$

In order to retain only the most relevant contribution in (40) three simplifications can be made. First of all, terms proportional to $\partial_{z} V_{\alpha k}(z)$ are irrelevant and thus can be neglected. (40) becomes after the change of variables $l \rightarrow l-k$

$$
V_{\alpha}(x) V_{\beta}(y)=\int_{k} \int_{l}: \mathrm{e}^{i(\alpha+\beta) \nabla r(z)+i l r(z)}: \mathrm{e}^{-\langle(\alpha \nabla+k) r(x)(\beta \nabla+l-k) r(y)\rangle_{0}}+\text { less singular terms }
$$

The integration over $l$ yields $\tilde{\delta}^{d}(r(z))$ plus its higher derivatives, which are irrelevant and thus neglected:

$$
\begin{array}{r}
V_{\alpha+\beta}(z) \int_{k} \mathrm{e}^{-\langle(\alpha \nabla+k) r(x)(\beta \nabla-k) r(y)\rangle_{0}}=V_{\alpha+\beta}(z) \int_{k} \mathrm{e}^{-k^{2}|x-y|^{4-D}-[\alpha(x-y)][\beta(x-y)](4-D)(2-D)|x-y|^{-D}} \\
\mathrm{e}^{-(4-D) \alpha \beta|x-y|^{2-D}+\left[\frac{\alpha+\beta}{2}(x-y)\right]^{2}(4-D)^{2}|x-y|^{-D}}
\end{array}
$$

where the integral over $k$ was shifted to isolate the term quadratic in $k$. After integration over $k$ equation (42) becomes:

$V_{\alpha+\beta}(z)\left(\frac{1}{|x-y|^{4-D}}\right)^{d / 2} \mathrm{e}^{\left[\frac{\alpha+\beta}{2}(y-x)\right]^{2}(4-D)^{2}|x-y|^{-D}-[\alpha(x-y)][\beta(x-y)](4-D)(2-D)|x-y|^{-D}-(4-D) \alpha \beta|x-y|^{2-D}}$

As explained in section 4 , the integration over the relative distance determines the renormalization of an operator. So we have to analyze the singularity for $x \rightarrow y$. Introducing the dimensional regularization parameter $\varepsilon$,

$$
\varepsilon=D-2 d+\frac{D d}{2}
$$

we get

$$
\left(\frac{1}{|x-y|^{4-D}}\right)^{d / 2}=|x-y|^{\varepsilon-D}
$$

Integration over $|x-y|$ thus yields pole terms in $\varepsilon$. In addition, the only dependence of the pole term on the exponential factors in (43) comes from $|x-y|=0$. In the spirit of analytic continuation we choose $D<2$ in order to have a regular expression for the exponential factors in (43). As by this way they equal 1 at $x=y$, the analytical continuation to $D \geq 2$ is unique, delivering 1 for the whole range. This would not be the case, if the limit $D \rightarrow 2$ had been performed before. 


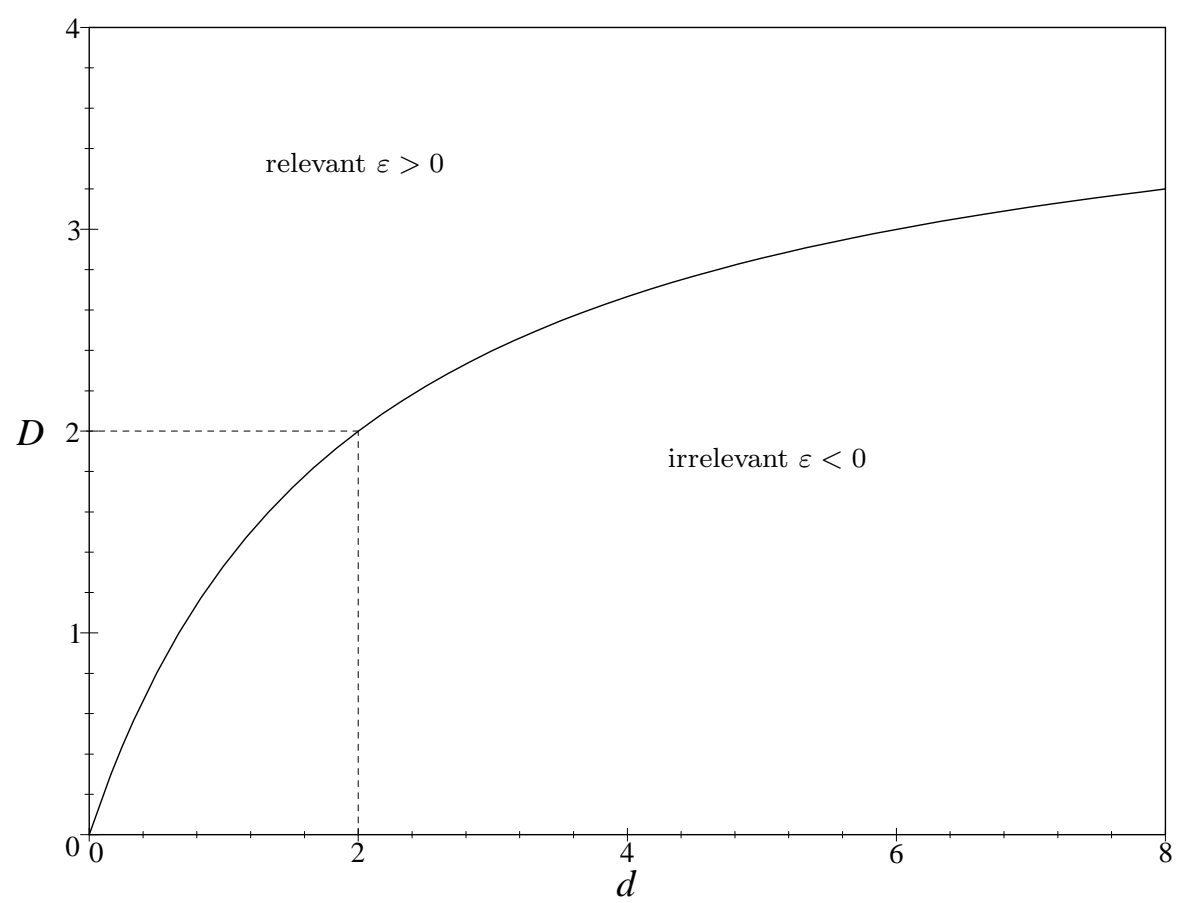

Figure 2: The critical curve $\varepsilon=0$

Finally we arrive at:

$$
\begin{aligned}
\int_{|x-y|<L} V_{\alpha}(x) V_{\beta}(y) & =V_{\alpha+\beta}(z) \int_{0}^{L} \frac{\mathrm{d} s}{s} s^{\varepsilon}+\text { less singular terms } \\
& =V_{\alpha+\beta}(z) \frac{L^{\varepsilon}}{\varepsilon}+\text { less singular terms }
\end{aligned}
$$

We construct now eigen-operators $E(x)$ of the contraction. Define

$$
E(x)=\int_{\alpha} e(\alpha) V_{\alpha}(x)
$$

which have to satisfy

$$
E(x) E(y)=|x-y|^{\varepsilon-D} E(z)+\text { less singular terms . }
$$

This fixes the normalization of $E(x)$. Plugging in the definition of $E(x)$ results in

$$
\int_{\alpha} \int_{\beta} e(\alpha) e(\beta) \delta(\gamma-\alpha-\beta)=e(\gamma)
$$

This equation can be solved by introducing the Fourier transform of $e(\alpha)$ :

$$
\tilde{e}(p)=\int_{\alpha} \mathrm{e}^{i p \alpha} e(\alpha)
$$

(49) becomes:

$$
\tilde{e}(p)^{2}=\tilde{e}(p)
$$


Let us recall that $\alpha$ was in $\mathbb{R}^{2} \times \mathbb{R}^{2}$, hence $p$.

Solutions of (51) are characteristic functions of (measurable) subsets $M$ of $\mathbb{R}^{2} \times \mathbb{R}^{2}$ :

$$
\tilde{e}(p)=\chi_{M}(p)
$$

Eigen-operators of the contraction (46) and therefore of the renormalization-group flow are:

$$
\begin{aligned}
E_{M}(x) & =\int_{\alpha} \int_{p} \mathrm{e}^{-i p \alpha+i \alpha \nabla r(x)} \chi_{M}(p) \tilde{\delta}^{d}(r(x)) \\
& =\chi_{M}(\nabla r(x)) \tilde{\delta}^{d}(r(x))
\end{aligned}
$$

Another interesting conclusion can be drawn: Rewriting (48) for two different perturbations $E_{M_{1}}(x)$ and $E_{M_{2}}(y)$ gives in the limit $|x-y| \rightarrow 0$

$$
E_{M_{1}}(x) E_{M_{2}}(y)=|x-y|^{\varepsilon-D} E_{M_{1} \cap M_{2}}\left(\frac{x+y}{2}\right)+\text { less singular terms } .
$$

This is an orthogonality relation for contractions.

At this point we should study what happens if in the free Hamiltonian (四) we do not introduce the factor $\frac{1}{2-D}$, i.e. if we use

$$
\tilde{\mathcal{H}}_{0}=-\frac{1}{2(4-D)} \int_{x} \frac{1}{2}(\Delta r(x))^{2}
$$

instead of $\mathcal{H}_{0}$. Equation (43) then becomes

$$
V_{\alpha+\beta}(z)\left(\frac{2-D}{|x-y|^{4-D}}\right)^{d / 2} \mathrm{e}^{\left[\frac{\alpha+\beta}{2}(y-x)\right]^{2} \frac{(4-D)^{2}}{2-D}|x-y|^{-D}-[\alpha(x-y)][\beta(x-y)](4-D)|x-y|^{-D}-\frac{4-D}{2-D} \alpha \beta|x-y|^{2-D}}
$$

This equation looks rather ugly, so let us put $\alpha=\beta=0$ for the moment. If $d \neq 2$ (56) is even non-analytic in the regularization parameter $D$ for $D \rightarrow 2$. But also the case $d=2$ is peculiar:

$$
(2-D) V_{0}(z) \cdot \frac{1}{|x-y|^{4-D}}
$$

Although the integration over $x-y$ yields a pole term in $1 /(2-D)$

$$
\int_{x} \frac{1}{|x|^{4-D}}=\int_{\Lambda} \frac{\mathrm{d} x}{x} x^{-2(2-D)}=\frac{1}{2(2-D)} \Lambda^{2(2-D)}
$$

it will be cancelled by the factor $(2-D)$ in (57). The system has no UV-divergence at all! For $\alpha \neq \beta$ the situation is even worse: Strong IR-singularities appear. We conclude that the Hamiltonian (55) is too "weak" and thus there is no way to define a sensible model in the limit $D \rightarrow 2$. 


\section{Interpretation of the result}

The operators $E_{M}(x)$ defined in (53) were constructed as eigen-oprators of the contraction, equation (48) or equivalently (20). Their renormalization has been analyzed in section 4 . There we showed that in the regime $\varepsilon>0$ the renormalization-group flow of $\lambda_{M}$ in

$$
\mathcal{H}_{M}=-\frac{1}{2(4-D)(2-D)} \int_{x} \frac{1}{2}(\Delta r(x))^{2}+\lambda_{M} \int_{x} E_{M}(x)
$$

has an IR-stable fixed point $\lambda_{M}^{*}=2 \varepsilon$. This result is independant of $M$ i.e. the fixed-point Hamiltonian is:

$$
\mathcal{H}^{*}=-\frac{1}{2(4-D)(2-D)} \int_{x} \frac{1}{2}(\Delta r(x))^{2}+2 \varepsilon \int_{x} \tilde{\delta}^{d}(r(x))
$$

So the interaction part of $\mathcal{H}^{*}$ does not depend on $\nabla r(x)$.

This result however may be false in practical cases. Suppose

$$
\mathcal{H}(t)=\mathcal{H}_{0}+\int_{p} f(t, p) \int_{x} E_{\{p\}}(x)
$$

where $f$ is normal distributed

$$
f(1, p)=2 \varepsilon \mathrm{e}^{-p^{2} / \sigma^{2}}
$$

and $\mu=t^{-1} \mu_{0}$.

The typical time $\tilde{t}$ which is necessary until $f(t, p)$ has reached the fixed point $2 \varepsilon$ is approximately

$$
\tilde{t}(p) \approx \frac{1}{\varepsilon} \frac{p^{2}}{\sigma^{2}},
$$

thus increases rapidly with $p$. If the microscopical Hamiltonian is given by (61) and (62) and if the microscopical scale and the scale of experiment are related by a renormalizationgroup transformation with say $t=10^{6}$, then the modes with $t^{*}>10^{6}$ will stay nearly 0 after the renormalization-group transformation. Stated otherwise, the critical regime for these modes is not reached. Whether this line of arguments is relevant depends on the initial values of $f(1, p)$.

\section{Conclusions}

We discussed a 2-dimensional field theory which is not conformal invariant but which can be treated in the framework of perturbation theory. Although the question of the physical interpretation of the model, especially the normalization involved in (田), had to stay open, a complete classification of all marginal impurity like perturbations was given at 1-loop order. Those are characteristic functions on $\mathbb{R}^{2} \times \mathbb{R}^{2}$. The renormalization-flow shows a rich structure which is special for the considered model.

\section{Acknowledgements}

It is a pleasure to thank François David, Stefan Kehrein, Sergey Shabanov, Jean ZinnJustin and Jean-Bernard Zuber for useful discussions and François David and Jean ZinnJustin for a careful reading of the manuscript. 


\section{References}

[1] F. David, B. Duplantier and E. Guitter, Nucl. Phys. B394 (1993) 555-664

[2] F. David, B. Duplantier and E. Guitter, Phys. Rev. Lett. 72 (1994) 311

[3] J. Polchinski, Nucl. Phys. B303 (1988) 226-236

[4] F. Ferrari, hep-th/9507142

[5] K. J. Wiese and F. David, Nucl. Phys. B450 (1995) 495-557

[6] J. Zinn-Justin, Quantum Field Theory and Critical Phenomena, Oxford 1989 